\title{
A mathematical model to study the COVID-19 pandemic in India
}

\author{
Agraj Tripathi $^{1}{ }^{1} \cdot$ Ram Naresh Tripathi $^{2} \cdot$ Dileep Sharma $^{3}$
}

Received: 17 June 2021 / Accepted: 5 September 2021 / Published online: 23 September 2021

(c) The Author(s), under exclusive licence to Springer Nature Switzerland AG 2021

\begin{abstract}
In this paper, a compartmental model is proposed to study the dynamics of COVID-19 pandemic caused by the coronavirus SARS-CoV-2 and the role of media in controlling this ongoing infection. Model includes implementation of media awareness as a control measure to mitigate the spread of the disease. In the proposed model, we have divided the total human population into four sub-classes, namely susceptibles, asymtomatic infectives, aware susceptibles and symptomatic infectives (or Isolated infectives which are under treatment/hospitalized) incorporating classes representing cumulative density of virus and media alert. The important mathematical features of the model are thoroughly investigated. The endemic equilibrium is found to be locally asymptotically stable as well as non-linearly asymptotically stable with certain conditions. Numerical simulations are also carried out in support of the analytical results and to show the effects of certain key parameters.
\end{abstract}

Keywords Coronavirus $\cdot$ Media $\cdot$ Immigration $\cdot$ Stability $\cdot$ Simulation

Mathematics Subject Classification 92D25 $\cdot 34 \mathrm{~A} 34 \cdot 34 \mathrm{M} 04$

\section{Introduction}

In present time, the coronavirus infection is the most common infection escalated worldwide with alarming rate. The spread of coronavirus disease 2019 (COVID-19) is threatening people's physical and mental health and even life safety. Almost every country is facing the impact of this dreaded disease. To contain the spread of the disease, countrywide or local lockdown was imposed which itself is enough to describe the seriousness of the pandemic. Globally, till 30

Agraj Tripathi

agrajtripathi@gmail.com

Ram Naresh Tripathi

rntripathi@hbtu.ac.in

Dileep Sharma

dilip_hbti@yahoo.com

1 Department of Basic Science and Humanities, Pranveer Singh Institute of Technology, Kanpur, Uttar Pradesh 209305, India

2 Department of Mathematics, School of Basic and Applied Sciences, Harcourt Butler Technical University, Kanpur, Uttar Pradesh 208002, India

3 Department of Mathematics, DBS (PG) College, Dehradun 248001, India
May 2021, there have been $169,597,415$ confirmed cases of COVID-19, including 3,530,582 deaths, reported to WHO. As of 26 May 2021, a total of 1,546,316,352 vaccine doses have been administered (Corona virus fact sheet by WHO 2021). Figures of COVID-19 cases clearly indicate the huge impact of the disease on human population. Therefore, it is essential to pay the attention to study the dynamics of this disease and suggest the ways to stop further its spreading.

This recent spread of COVID-19 disease has led to many theoretical investigations suggesting various non-pharmaceutical interventions (Aldila et al. 2020; Almomani and AlQuran 2020; Agaba 2020; Cai et al. 2020; Chen et al. 2020; Chekol and Melesse 2020; Contreras et al. 2020; Cooper et al. 2020; Corbet et al. 2021; Cui et al. 2008a, b; Dubey et al. 2016; Fredj and Cherif 2020; Gao et al. 2020; $\mathrm{Hu}$ et al. 2020; Khan and Atangana 2020; Krishna and Prakash 2020; Misra et al. 2015; Mitchel 2020; Naresh et al. 2009, 2011a; Kumar and Somani 2020; Pai et al. 2020; Panovska-Griiths 2020; Pandey et al. 2020; Sarkar et al. 2020; Shereen et al. 2020; Wang et al. 2020; Wiah et al. 2020; Zeb et al. 2020; Zehra et al.2020). In particular, Aldila et al. (2020) formulated a modified susceptible, exposed, infectious, recovered compartmental model considering asymptomatic individuals. They used the incidence data from the city of Jakarta, Indonesia and observed that the strict social 
distancing is very much helpful is delaying the outbreak of the disease. However, if the strict social distancing policy is relaxed, a massive rapid-test intervention should be conducted to avoid a large-scale outbreak in future. Contreras et al. (2020) developed a general multi-group SEIRA model for representing the spread of COVID-19 among a heterogeneous population. Cooper et al. (2020) studied the effectiveness of the modeling approach on the pandemic due to the spreading of the novel COVID-19 disease and developed a susceptible-infected-removed (SIR) model that provides a theoretical framework to investigate its spread within a community. Liu et al. (2020) developed models to account for latency period and evaluated the role of the exposed or latency period on the dynamics of a COVID-19. Sarkar et al. (2020) proposed a compartmental model that predicts the dynamics of COVID-19 in 17 provinces of India and the overall India. Almomani and AlQuran (2020) observed that with the spread of the coronavirus globally, the negative effects increased at all levels especially in the economic and social sectors. Their study shows that the situation further worsened by the spread of rumors and false information about the disease circulated through social media and online platforms. Cai et al. (2020) observed in their study, the impact on mental health, resilience and social support of health care workers due to continuously being in close vicinity with persons affected with COVID-19. Chen et al. (2020) proposed a reservoir-people transmission network model for calculating the transmissibility of SARS-CoV-2. Fredj and Cherif (2020) proposed a deterministic compartmental model based on the clinical progression of the disease, the epidemiological state of the individuals and the intervention for the dynamics of COVID-19 infection. Khan and Atangana (2020) proposed a mathematical model by assuming interaction among the bats and unknown hosts, then among the peoples and the infection reservoir (seafood market). The seafood markets, considered to be the main source of infection, where bats and the unknown hosts (may be wild animals) leave the infection for further transmission. Krishna and Prakash (2020) developed a phase-based mathematical model for COVID-19 and concluded that the spreading of COVID-19 capacity is superior than MERS into the Middle East nationals.

The disease spread is faster in closed population groups through small droplets emanated from the nose or mouth via coughing, sneezing or exhalation of a COVID-19-affected person. These droplets may deposit on objects and surfaces around the person or remain airborne for some time. The susceptibles may get exposed to COVID-19 infection upon touching their eyes, nose or mouth after coming in contact with these objects or surfaces or if they breathe in droplets from a person with COVID-19 who coughs out or exhales droplets. From the above studies, it may be noted that these investigations ignore a very important prevention strategy, i.e. media awareness campaign. Though the vaccine has been developed but it may take years to reach the entire population. Thus, it would be very effective and helpful to educate the people about the COVID-19, its causes and preventive measures in the form of non-pharmaceutical intervention strategies like face cover/mask wearing, social distancing, etc. through media awareness campaigns. Media awareness campaigns not only play a very crucial role in creating awareness to reduce the spread of any infectious disease, but also induce the behavioral change in general population which ultimately changes the pattern of disease spread (Cui et al. 2008a, b; Misra et al. 2011, 2015; Naresh et al. 2011a, b; Tripathi et al. 2007; Tripathi and Naresh 2019). The transmission dynamics of Corona virus is given in Fig. 1.

\section{Formulation of mathematical model}

We consider the population of size $N(t)$ at time $t$ with constant immigration rate $A$. The population size $N(t)$ is divided into four subclasses of susceptibles $X(t)$, asymptomatic infectives $Y(t)$ (also assumed to be infectious), aware susceptibles (who are staying home/isolated) $X_{M}(t)$ and symptomatic infectives $Y_{i}(t)$ (who are isolated for treatment/hospitalized). The variable $V(t)$ be the cumulative density of virus and the variable $M(t)$ denotes the cumulative density representing media alert to make the

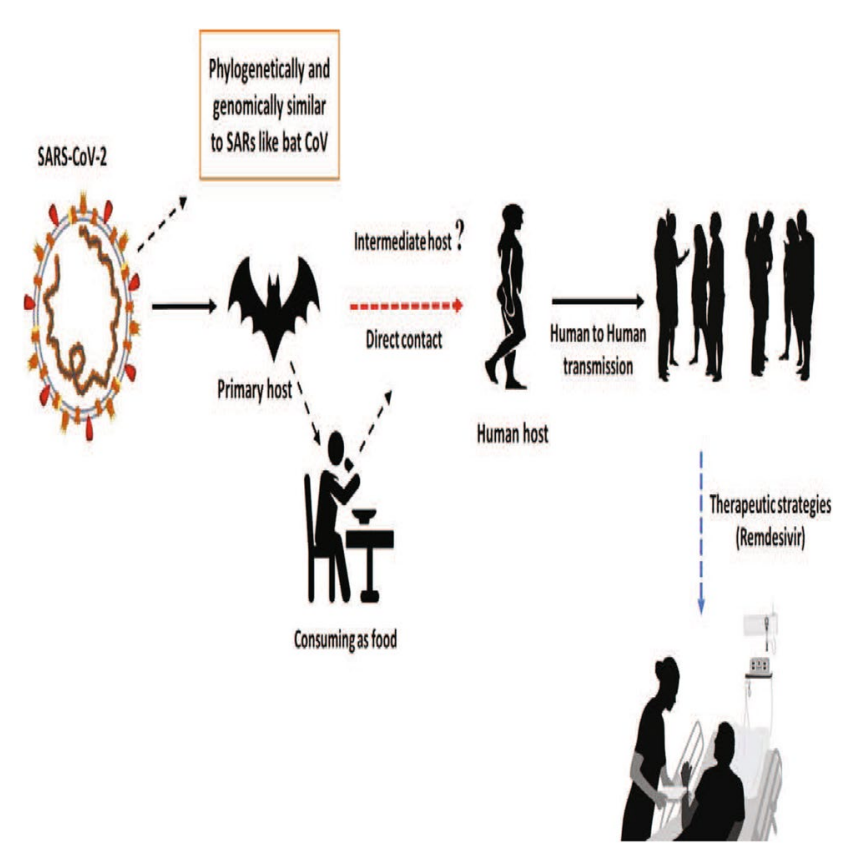

Fig. 1 Schematic diagram of transmission dynamics of Corona virus (Shereen et al. 2020) 
population aware of the disease (Misra et al. 2011). With these considerations, the mathematical model is proposed as follows,

$$
\begin{aligned}
& \frac{\mathrm{d} X}{\mathrm{~d} t}=A-\beta \frac{X Y}{N}-\lambda V X-\nu \frac{X M}{1+\gamma M} \\
& -\mathrm{d} X+\lambda_{11} X_{M}+\beta_{11} Y_{i}+\alpha Y \\
& \frac{\mathrm{d} Y}{\mathrm{~d} t}=\beta \frac{X Y}{N}+\lambda V X-\mathrm{d} Y-\alpha Y-\beta_{1} Y \\
& \frac{\mathrm{d} X_{M}}{\mathrm{~d} t}=\nu \frac{X M}{1+\gamma M}-\mathrm{d} X_{M}-\lambda_{11} X_{M} \\
& \frac{\mathrm{d} Y_{i}}{\mathrm{~d} t}=\beta_{1} Y-\mathrm{d} Y_{i}-\alpha_{1} Y_{i}-\beta_{11} Y_{i} \\
& \frac{\mathrm{d} V}{\mathrm{~d} t}=\theta Y-\theta_{0} V \\
& \frac{\mathrm{d} M}{\mathrm{~d} t}=\mu Y-\sigma M
\end{aligned}
$$

where $d$ is the natural mortality rate constant, $\alpha_{1}$ is diseaseinduced death rate constant. The constant $\beta$ represents the transmission rate by which susceptible individuals $X(t)$ come into contact with asymptomatic infectives $Y(t)$ directly and become infective. The constant $\lambda$ represents the transmission rate by which susceptible individuals $X(t)$ become infected by directly coming in contact with virus $V(t)$ deposited on surfaces/objects or airborne droplets. Some of the aware susceptibles may again become susceptible with a rate $\lambda_{11}$ due to fading of the effect of media awareness campaigns. The term $\frac{v M}{1+\gamma M}$ denotes the effect of media coverage on susceptible population where $v$ can be thought of as the dissemination rate of awareness programs among susceptibles and $\gamma$ limits the effect of awareness programs on susceptibles, $\frac{v}{\gamma}$ is the maximum effect that media can put on susceptibles (Dubey et al. 2016). The constant $\alpha$ is the rate by which mild infectives from asymptomatic class are cured but remain vulnerable to join the susceptible class and $\beta_{1}$ is the rate of appearance of symptoms by which asymptomatic infectives join the symptomatic (hospitalized/isolated) class. The constant $\beta_{11}$ represents the recovery rate coefficient of symptomatic (hospitalized/isolated) individulas who again become susceptible after recovery. The viral density $V(t)$ is assumed to be directly proportional to the asymptomatic infectives where $\theta$ is the rate of increase of $V$. The constant $\theta_{0}$ is the rate by which viral density declines due to control/preventive measures. The constant $\mu$ represents the rate by which awareness programs are being implemented and are assumed to be directly proportional to the asymptomatic infective population. The constant $\sigma$ represents the depletion rate of these programs due to ineffectiveness, social problems in the population, etc.

Since $N(t)=X(t)+Y(t)+X_{M}(t)+Y_{i}(t)$, the model system (1)-(6) can be rewritten as,

$\frac{\mathrm{d} N}{\mathrm{~d} t}=A-\mathrm{d} N-\alpha_{1} Y_{i}$

$\frac{\mathrm{d} Y}{\mathrm{~d} t}=\beta \frac{\left(N-Y-X_{M}-Y_{i}\right) Y}{N}$

$$
+\lambda V\left(N-Y-X_{M}-Y_{i}\right)-\left(d+\alpha+\beta_{1}\right) Y
$$

$\frac{\mathrm{d} X_{M}}{\mathrm{~d} t}=v \frac{\left(N-Y-X_{M}-Y_{i}\right) M}{1+\gamma M}-\mathrm{d} X_{M}-\lambda_{11} X_{M}$

$\frac{\mathrm{d} Y_{i}}{\mathrm{~d} t}=\beta_{1} Y-\left(d+\alpha_{1}+\beta_{11}\right) Y_{i}$

$\frac{\mathrm{d} V}{\mathrm{~d} t}=\theta Y-\theta_{0} V$

$\frac{\mathrm{d} M}{\mathrm{~d} t}=\mu Y-\sigma M$

with initial conditions, $N(0)=N_{0}>0, Y(0)=Y_{0} \geq 0$, $X_{M}(0)=X_{M 0} \geq 0, Y_{i}(0)=Y_{i 0} \geq 0, V(0)=V_{0} \geq 0$, and $M(0)=M_{0} \geq 0$

\section{Invariant region}

To study the stability of equilibrium points, we need the bounds of dependent variables of the model system (7)-(12). For this, we find the invariant region in the form of following lemma, stated without proof.

Lemma The closed set $D=\left\{\left(N, Y, X_{M}, Y_{i}, V, M\right) \in R_{6}^{+}\right.$: $\left.\frac{A}{\alpha_{1}+d}<N<\frac{A}{d}, 0<Y+X_{M}+Y_{i} \leq N, 0 \leq V \leq \frac{\theta}{\theta_{0}} \frac{A}{d}, 0 \leq M \leq \frac{\mu}{\sigma} \frac{A}{d}\right\}$

\section{Existence of equilibria}

The system (7)-(12) exhibits two equilibria, namely

(i) $E_{0}\left(\frac{A}{d}, 0,0,0,0,0\right)$, the disease-free equilibrium, which is obvious.

(ii) $E^{*}\left(N^{*}, Y^{*}, X_{M}^{*}, Y_{i}^{*}, V^{*}, M^{*}\right)$, the endemic equilibrium,

where $N^{*}, Y^{*}, X_{M}^{*}, Y_{i}^{*}, V^{*}$ and $M^{*}$ are the positive solutions which we get on solving the following system of algebraic equations obtained by putting right-hand side of model equations (7)-(12) to zero, 
$A-\mathrm{d} N-\alpha_{1} Y_{i}=0$

$\left(\frac{\beta Y}{N}+\lambda V\right)\left(N-Y-X_{M}-Y_{i}\right)$

$-\left(d+\alpha+\beta_{1}\right) Y=0$

$\nu \frac{\left(N-Y-X_{M}-Y_{i}\right) M}{1+\gamma M}-\mathrm{d} X_{M}-\lambda_{11} X_{M}=0$

$\beta_{1} Y-\left(d+\alpha_{1}+\beta_{11}\right) Y_{i}=0$

$\theta Y-\theta_{0} V=0$

$\mu Y-\sigma M=0$

From Eqs. (13), (17) and (18) we get, respectively,

$Y_{i}=\frac{A-\mathrm{d} N}{\alpha_{1}}=f(N)($ say $)>0$

$V=\frac{\theta}{\theta_{0}} Y>0$

$M=\frac{\mu}{\sigma} Y>0$

$Y=\frac{(A-\mathrm{d} N)\left(d+\alpha_{1}+\beta_{11}\right)}{\alpha_{1} \beta_{1}}=u f(N)>0$

here, $u=\frac{\left(d+\alpha_{1}+\beta_{11}\right)}{\beta_{1}}$, and from Eq. (15) we get,

$X_{M}=\frac{v[N-u f(N)-f(N)]}{v+\left(\lambda_{11}+d\right)\left[1+\gamma \frac{\mu}{\sigma} u f(N)\right]}=g(N)($ say $)$

Now on substituting all the values in Eq. (14) we get,

$$
\begin{aligned}
F(N)= & \left(\beta+\lambda \frac{\theta}{\theta_{0}} N\right)[N-u f(N)-g(N)-f(N)] \\
& -\left(\alpha+d+\beta_{1}\right) N=0
\end{aligned}
$$

It would be sufficient if we show that the Eq. (24) has one and only one positive root between $\frac{A}{\alpha_{1}+d}$ and $\frac{A}{d}$. To prove this, from Eq. (24) we have,

$$
\begin{aligned}
& F\left(\frac{A}{\alpha_{1}+d}\right)=-\frac{A}{\alpha_{1}+d}\left(\beta+\lambda \frac{\theta}{\theta_{0}} \frac{A}{\alpha_{1}+d}\right) \\
& {\left[u \frac{\left(\lambda_{11}+d\right)\left[1+\gamma \frac{\mu}{\sigma} u \frac{A}{\alpha_{1}+d}\right]}{v+\left(\lambda_{11}+d\right)\left[1+\gamma \frac{\mu}{\sigma} u \frac{A}{\alpha_{1}+d}\right]}\right]} \\
& -\left(\alpha+d+\beta_{1}\right) \frac{A}{\alpha_{1}+d}<0
\end{aligned}
$$

$F\left(\frac{A}{d}\right)=\frac{A}{d}\left(d+\alpha+\beta_{1}\right)\left[R_{0} \frac{\lambda_{11}+d}{v+\lambda_{11}+d}-1\right]>0$

provided $R_{0}\left(\frac{\lambda_{11}+d}{v+\lambda_{11}+d}\right)>1$. Here, $R_{0}$ represents the reproductive number, given as $R_{0}=\frac{\left(\beta+\lambda \frac{\theta}{\theta_{0}} \frac{A}{d}\right)}{\left(\alpha+d+\beta_{1}\right)}$.

Also, from Eq. (24) and after some algebraic manipulation,

$$
\begin{aligned}
F^{\prime}(N)= & 2 \beta+\lambda \frac{\theta}{\theta_{0}} N+\lambda \frac{\theta}{\theta_{0}} \\
& {[N-u f(N)-g(N)-f(N)]-2\left(\alpha+d+\beta_{1}\right) }
\end{aligned}
$$

since, $N-Y-X_{M}-Y_{i}=X$, therefore,

$F^{\prime}(N)=2 \beta+\lambda \frac{\theta}{\theta_{0}}(N+X)-2\left(\alpha+d+\beta_{1}\right)>0$

provided

$2 \beta+\lambda \frac{\theta}{\theta_{0}}(N+X)>2\left(\alpha+d+\beta_{1}\right)$

This shows that $F(N)=0$ has exactly one $\operatorname{root}\left(\right.$ say $N^{*}$ ) between $\frac{A}{\alpha_{1}+d}$ and $\frac{A}{d}$. Using $N^{*}$, the values of $Y^{*}, X_{M}^{*}, Y_{i}^{*}, V^{*}$ and $M^{*}$ can be found easily.

\section{Stability of equilibria}

In this section, we carry out the stability analysis of the equilibrium points. For this, the following theorems are proposed.

Theorem 4.1 The disease-free equilibrium $E_{0}$ is locally asymptotically stable if $R_{0}<1$ so that the infection does 
not persist in the population and under this condition the endemic equilibrium $E^{*}$ does not exist. It is unstable for $R_{0}>1$ and endemic equilibrium $E^{*}$ appears.

Theorem 4.2 The endemic equilibrium $E^{*}$ is locally asymptotically stable under the following conditions,

$20 a r<q\left(a+\lambda_{11}+d\right)$

$5 s \theta<r \theta_{0}$

$\frac{9}{4} \frac{\alpha_{1}^{2}}{d\left(\alpha_{1}+d+\beta_{11}\right)}<\frac{4}{15} \frac{d q r}{p^{2} \beta_{1}}$

See Appendix 1 for proof of Theorems 4.1 and 4.2.

Theorem 4.3 The endemic equilibrium $E^{*}$ is non-linearly asymptotically stable under the following conditions,

$20 a<\left(a+\lambda_{11}+d\right)$

$5 \theta \lambda \frac{A}{d}<r \theta_{0}$

$\frac{9}{4} \frac{\alpha_{1}^{2}}{d\left(\alpha_{1}+d+\beta_{11}\right)}<\frac{4}{15} \frac{d r^{2}}{Y^{* 2} \beta_{1} \phi}$

See Appendix 2 for proof of Theorem 4.3.

The symbols used in Theorems 4.2 and 4.3 are defined as follows:
$p=\frac{\beta\left(Y^{*}+X_{M}^{*}+Y_{i}^{*}\right) Y^{*}}{N^{*} 2}+\lambda V^{*}>0, \quad q=\frac{\beta Y^{*}}{N^{*}}+\frac{\lambda V^{*}\left(N^{*}-X_{M}^{*}-Y_{i}^{*}\right)}{Y^{*}}>0$, $r=\frac{\beta Y^{*}}{N^{*}}+\lambda V^{*}>0, \quad s=\lambda\left(N^{*}-Y^{*}-X_{M}^{N^{*}}-Y_{i}^{*}\right)>0$, $a=\frac{N_{\nu M^{*}}^{*}}{1+\gamma M^{*}}>0,{ }_{b}=\frac{v\left(N^{*}-Y^{*}-X_{M}^{*}-Y_{i}^{*}\right)}{\left(1+\gamma M^{*}\right)^{2}}>0, \phi=\left[\frac{\left(\alpha_{1}+d\right)}{d} \frac{\beta}{N^{*}}+\frac{\lambda V^{*}}{Y^{*}}\right]^{2}>0$

\section{Numerical analysis and discussion}

In this section, we perform some numerical simulations for the model system (7)-(12). For this, we integrate the system by fourth-order Runge-Kutta method using MATLAB. Most of the parameter values used in simulation are adopted from previously published articles, while others are estimated intuitively. The unit of parameters is in per day. We use initial values for simulation as stated below (Sarkar et al. 2020; Tripathi and Naresh 2019).

$N(0)=1300000000, Y(0)=0, X_{M}(0)=0$,

$Y_{i}(0)=1, V(0)=1, M(0)=0$

In Table 2, the reported corona-positive cases (confirmed infectives) in India are depicted during first wave of pandemic for the months January to August 2020. Till 18 September 2020, Indian government declared the total $5,214,677$ corona-positive cases, in which $4,112,551$ were cured/discharged and 8472 deaths were due to this disease. The active cases of corona were $1,017,754$. According to government of India, the recovery rate was $78.8 \%$ and death rate was $2.25 \%$ (Corona virus statistics by India 2020).

The parameters used in the model are given in Table 1.

From Fig. 2, we can see the trend of data as reported in Table 2 and the curve represented by the model for corona cases using the above parameters. It is noted that the number of confirmed infected persons (symptomatic/isolated)

Table 1 Parameters of the model

\begin{tabular}{ll}
\hline Parameter symbol & Value \\
\hline Recruitment rate in susceptible class $(A)$ & 10,000 [estimated] \\
Transmission rate of susceptibles to asymptomatic infectives class $(\beta)$ & 0.86 per day (Sarkar et al. 2020) \\
Transmission rate of susceptibles via coronavirus $(\lambda)$ & 0.00009 per day [estimated] \\
Mortality rate (days) & 0.000038 per day (Corona virus statistics by India 2020) \\
Recovery rate $\left(\beta_{11}\right)$ & 0.0036 per day (Corona virus statistics by India 2020) \\
Rate of appearance of symptoms $\left(\beta_{1}\right)$ & 0.14 per day [estimated] \\
Death rate in symptomatic infectives class $\left(\alpha_{1}\right)$ & 0.00011 per day (Corona virus statistics by India 2020) \\
Transfer rate of mild infectives to susceptible class $(\alpha)$ & 0.01 per day [estimated] \\
Efficacy of awareness programs $\left(\lambda_{11}\right)$ & 0.042 per day [estimated] \\
Rate of increase of viral density $(\theta)$ & $2 \times 10^{-7}$ g [estimated] \\
Depletion rate of viral density $\left(\theta_{0}\right)$ & $10^{-13} \mathrm{~g}$ [estimated] \\
Dissemination rate of awareness programs $(\nu)$ & 0.023 per day [estimated] \\
Limiting factor of awareness programs $(\gamma)$ & 1 (Tripathi and Naresh 2019) \\
Implementation rate of media awareness programs $(\mu)$ & 0.0068 [estimated] \\
Depletion rate of media programs due to ineffectiveness $(\sigma)$ & 0.001 [estimated] \\
\hline
\end{tabular}


Table 2 Reported corona positive cases during first wave of pandemic by months Jan. to Aug. 2020 (Corona virus statistics by India 2020)

\begin{tabular}{lllllllll}
\hline Months & Jan. & Feb. & Mar. & Apr. & May & Jun. & Jul. & Aug. \\
\hline Cases & 1 & 3 & 1071 & 33,050 & 182,143 & 566,840 & $1,638,870$ & $3,542,733$
\end{tabular}

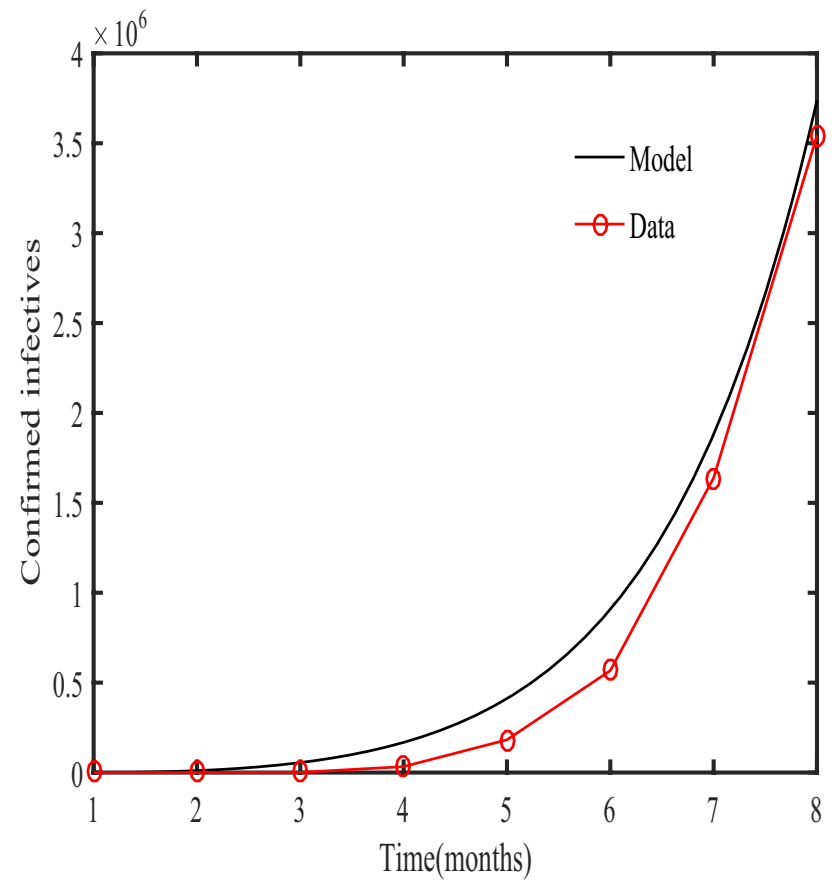

Fig. 2 Validation of model with Indian data

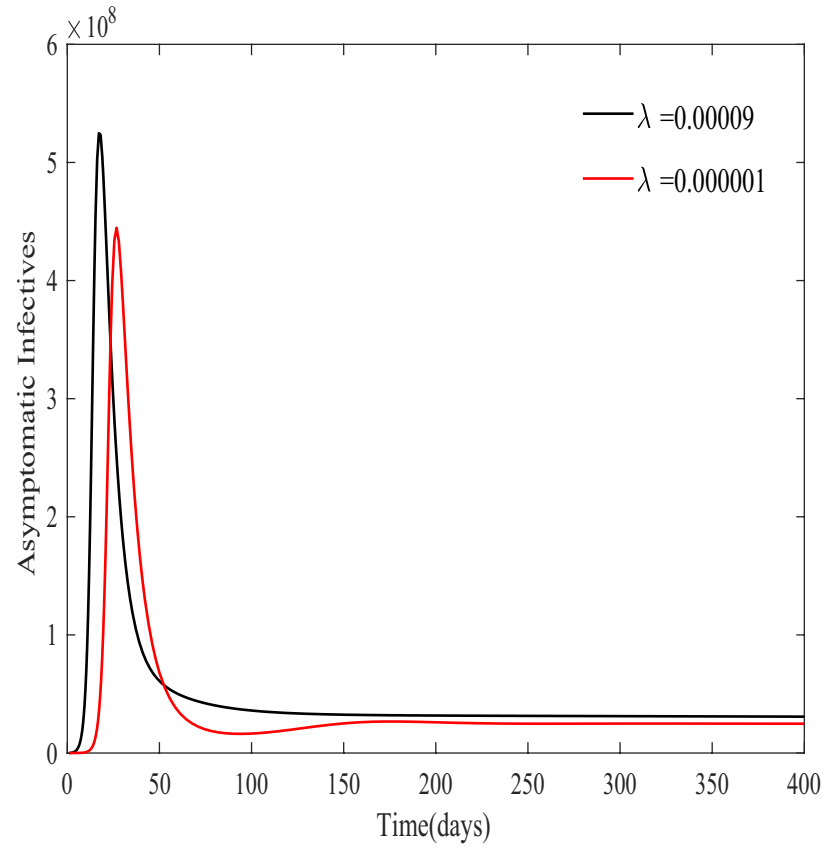

Fig. 3 Variation of asymptomatic Infectives $Y$ with time $t$ for different values of $\lambda$

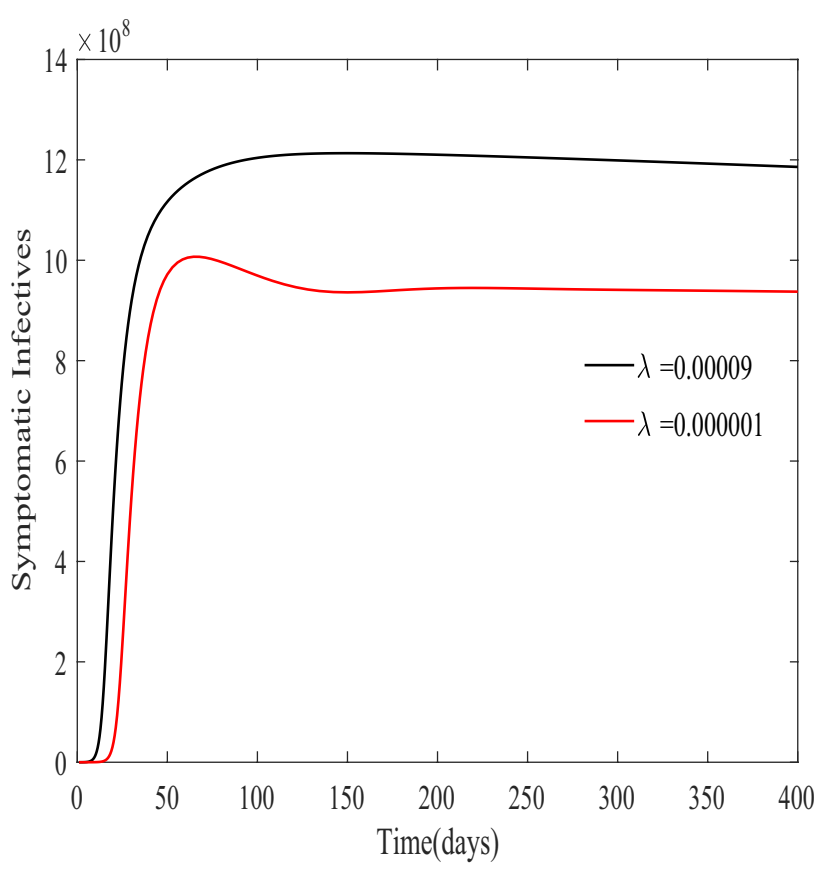

Fig. 4 Variation of symptomatic infectives $Y_{i}$ with time $t$ for different values of $\lambda$

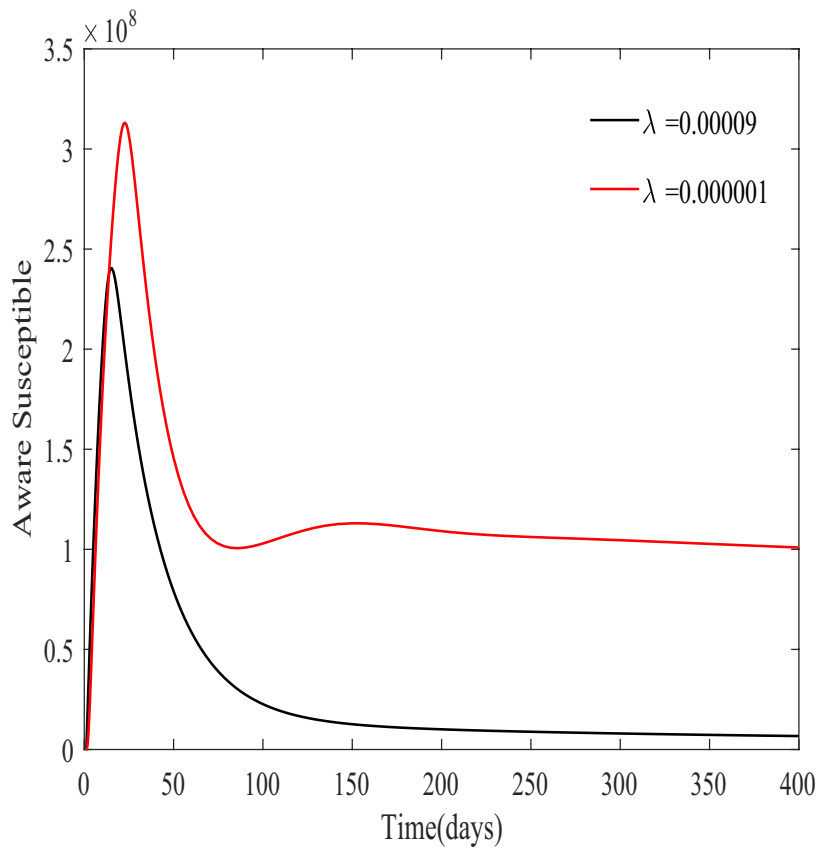

Fig. 5 Variation of aware susceptibles $X_{M}$ with time $t$ for different values of $\lambda$ 


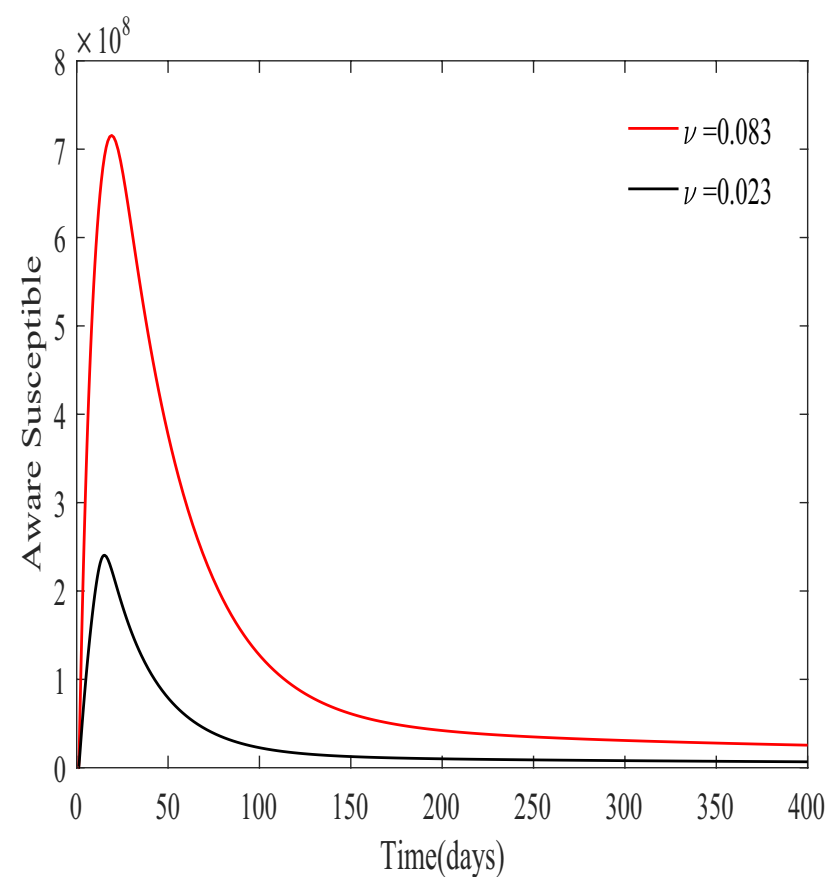

Fig. 6 Variation of aware susceptibles $X_{M}$ with time $t$ for different values of $v$

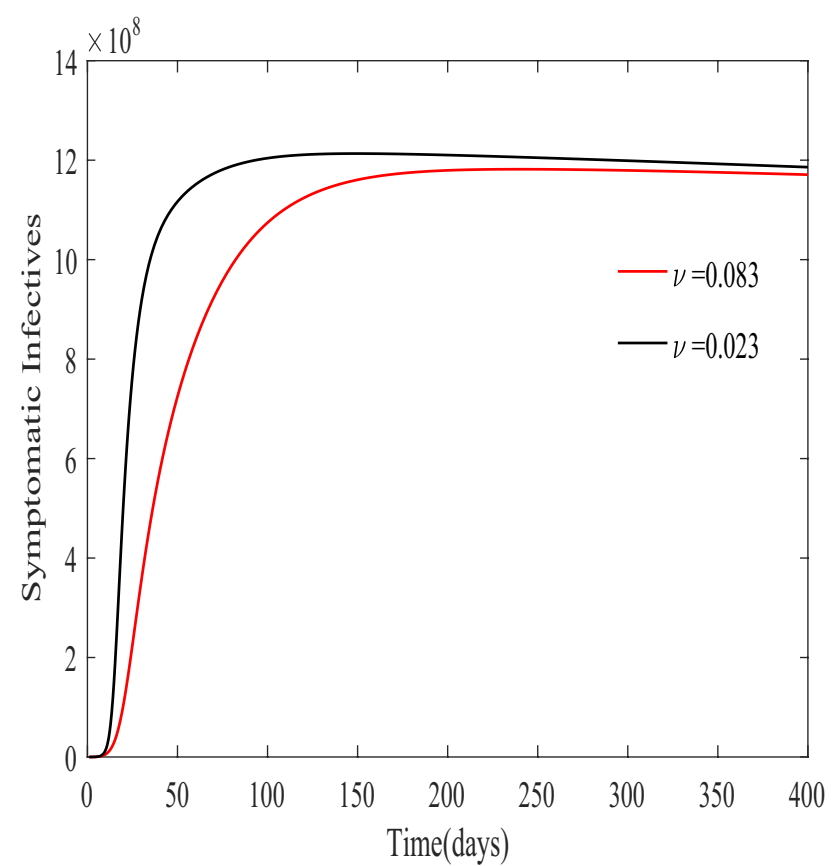

Fig. 7 Variation of symptomatic infectives $Y_{i}$ with time $t$ for different values of $v$

estimated by the model for the above set of parameter values is closer to the reported Indian data (Table 2).

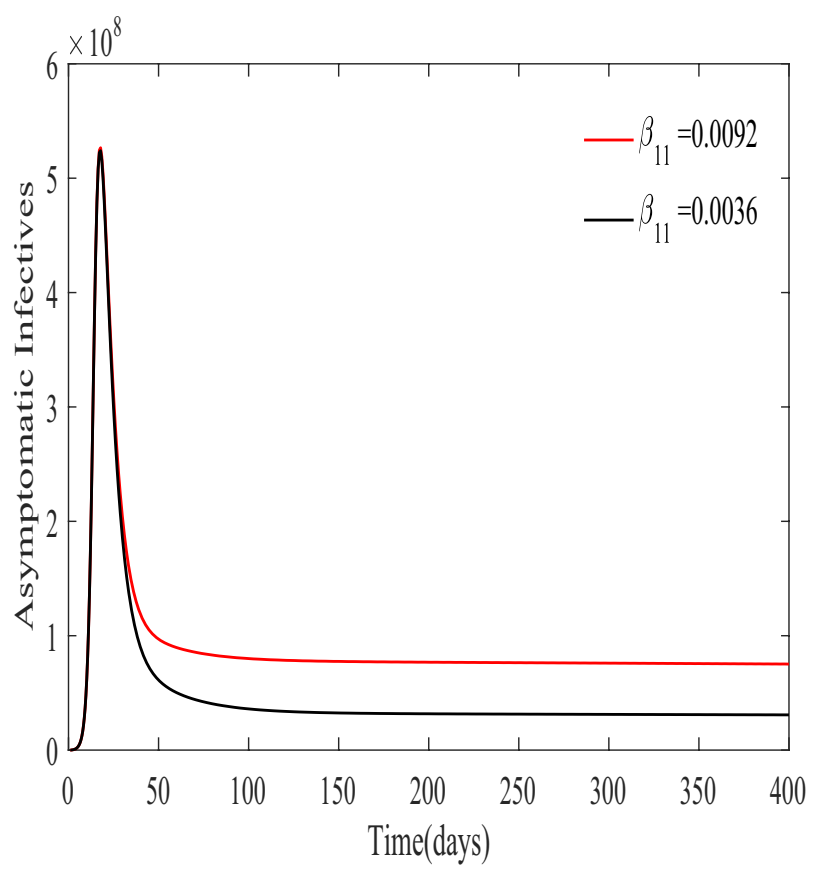

Fig. 8 Variation of asymptomatic Infectives $Y$ with time $t$ for different values of $\beta_{11}$

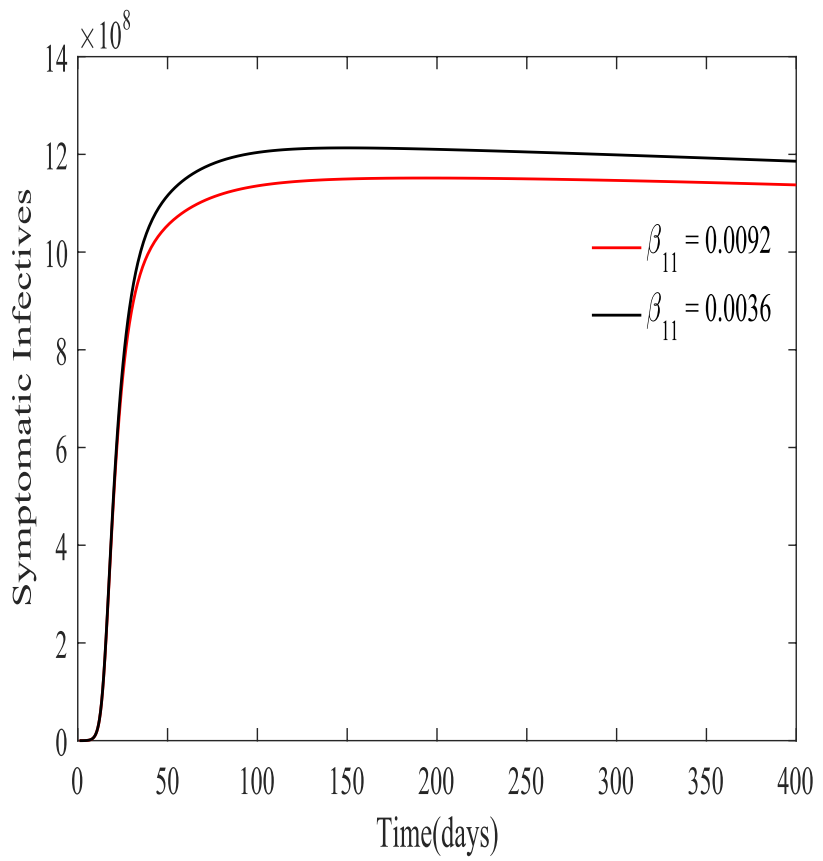

Fig. 9 Variation of symptomatic infectives $Y_{i}$ with time $t$ for different values of $\beta_{11}$

The results of numerical simulation are displayed graphically in Figs. 3, 4, 5, 6, 7, 8, 9, 10, 11, 12, 13 and 14. In Figs. 3 and 4, the behaviour of asymptomatic infectives and symptomatic infectives is shown with time for 


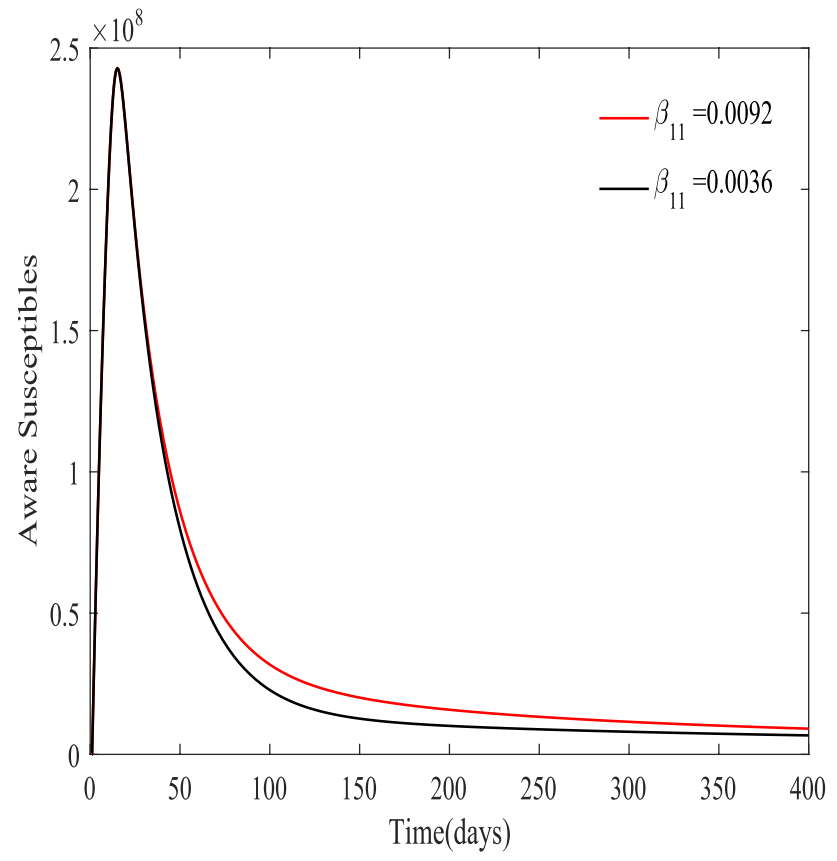

Fig. 10 Variation of aware susceptibles $X_{M}$ with time $t$ for different values of $\beta_{11}$

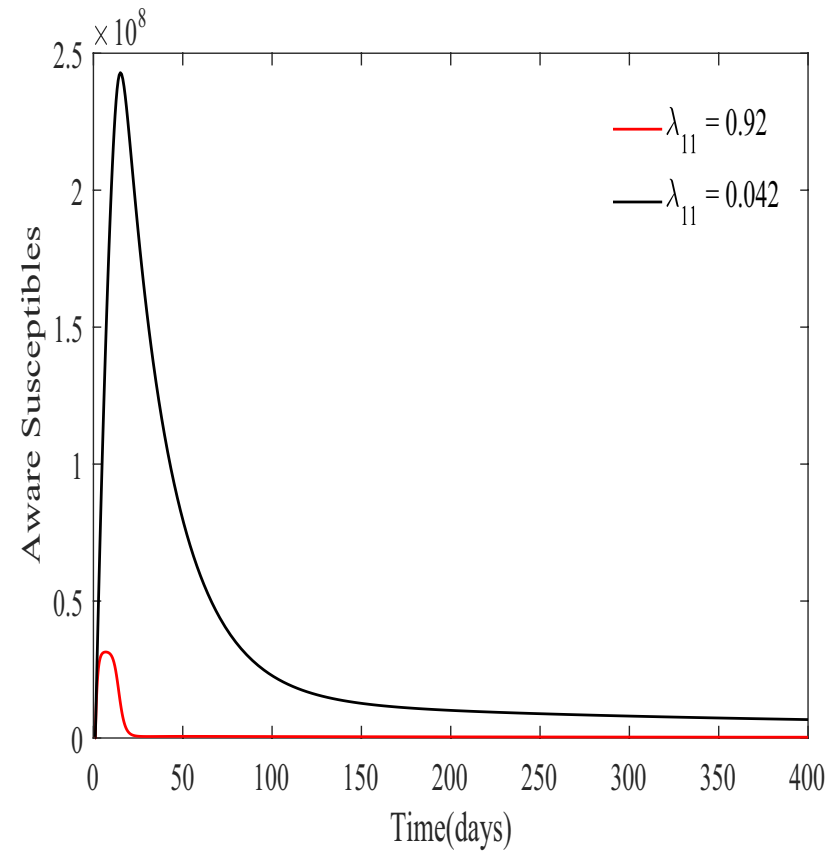

Fig. 11 Variation of aware susceptibles $X_{M}$ with time $t$ for different values of $\lambda_{11}$

different values of the transmission rate $\lambda$ due to direct contact of susceptibles with virus. It is seen from these figures that the number of asymptomatic infectives increases with increase in the transmission rate $\lambda$ due to direct contact of susceptibles with virus, (Fig. 3). Consequently,

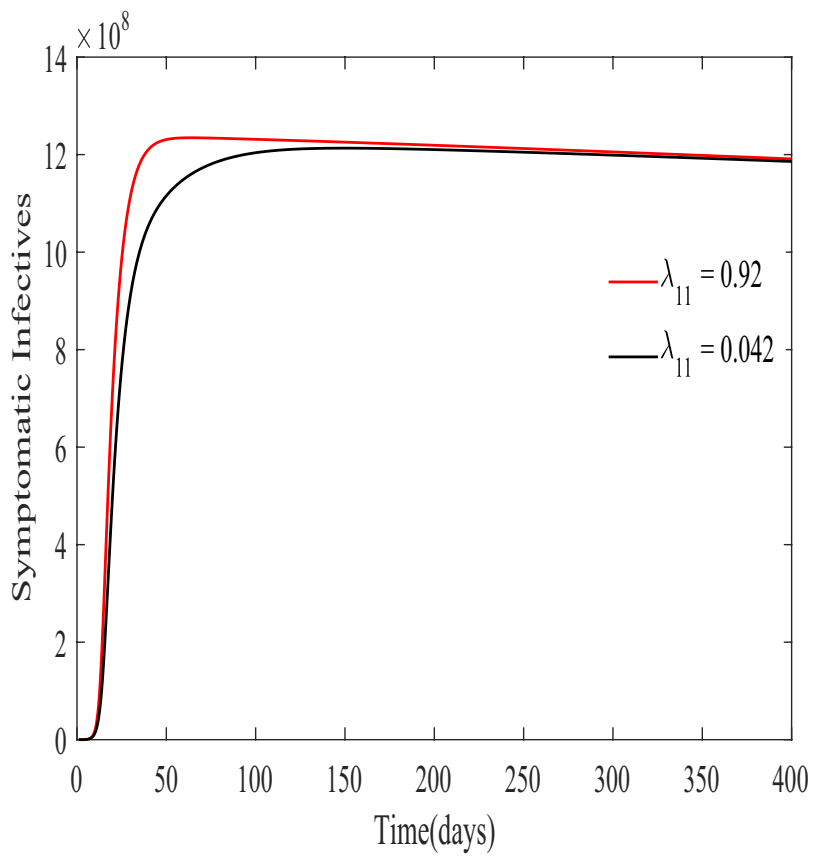

Fig. 12 Variation of symptomatic infectives $Y_{i}$ with time $t$ for different values of $\lambda_{11}$

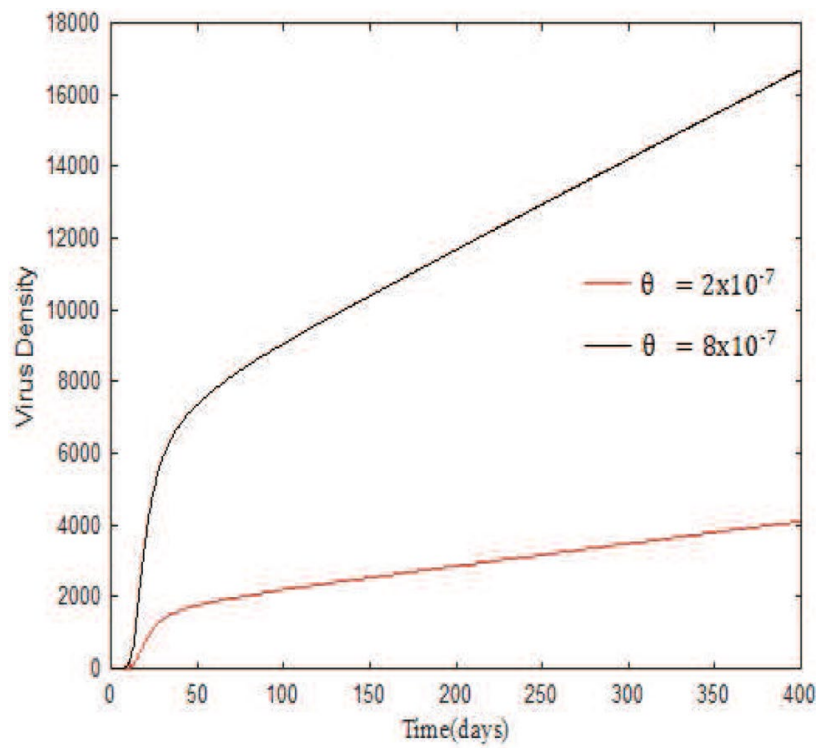

Fig. 13 Variation of virus density $V$ with time $t$ for different values of $\theta$

the number of symptomatic infectives also increases and remains endemic with higher viral load, (Fig. 4). This indicates that if the transmission rate is curtailed by way of face cover/mask wearing, social distancing, avoidance of crowded places etc., the number of asymptomatic infectives and consequently the symptomatic infectives can be decreased due to non-exposure with virus. In Fig. 5, we 


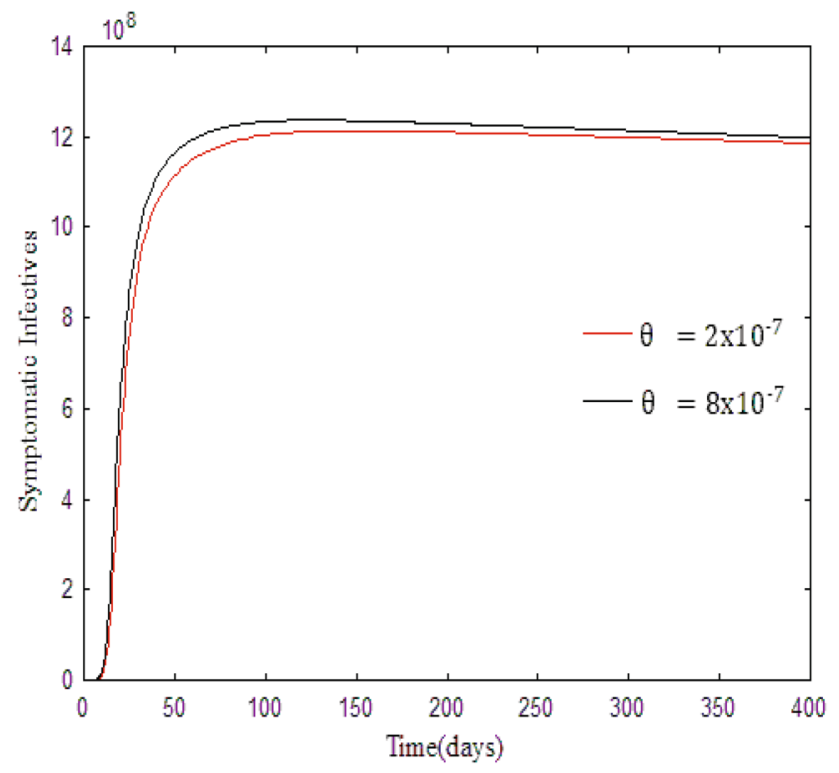

Fig. 14 Variation of symptomatic infectives $Y_{i}$ with time $t$ for different values of $\theta$

have shown the variation of aware susceptible population with time for different values of $\lambda$, the transmission rate due to direct contact of susceptibles with virus. It is noted from this figure that the aware susceptible population declines as the transmission rate $\lambda$ due to direct contact of susceptibles with virus increases.

In Figs. 6 and 7, the variation of aware susceptible population and symptomatic infectives is shown with time for different values of $v$, the dissemination rate of media awareness programs. It is observed that the aware susceptible population increases with increase in the dissemination rate of media awareness programs, (Fig. 6) and consequently the symptomatic infectives population decreases, (Fig. 7). This highlights the importance of media awareness campaigns as a result of which more and more susceptible persons become aware of disease escalation. The resulting behavioral change helps in reducing the spread of COVID-19 infection and hence the symptomatic population decreases.

In Fig. 8, the variation of asymptomatic infectives with time $t$ is shown for different values the recovery rate of symptomatic (hospitalized/isolated) infectives $\beta_{11}$. It is seen that the population of asymptomatic infectives increases in the long run with increase in the recovery rate of symptomatic infectives. These recovered symptomatic infectives remain susceptible to increase the susceptible population which in turn increases the asymptomatic infective population. The increase in the recovery rate of symptomatic (hospitalized/isolated) individuals $\beta_{11}$ decreases the population of symptomatic class, (Fig. 9). This recovered population of symptomatic individuals who are discharged from hospitals increases the susceptible population which makes the aware susceptible population to increase, (Fig. 10).

In Figs. 11 and 12, the variation of aware susceptible population and symptomatic (hospitalized/isolated) infective population with time $t$ is shown for different values of $\lambda_{11}$, the rate at which aware susceptibles lose the effect of awareness and become susceptible again due to fading of the effect of media programs. It is seen from these figures that as the effect of media awareness programs fades away, the aware susceptible population declines, (Fig. 11) and consequently the symptomatic infectives population increases, (Fig. 12). Thus, if aware susceptible population loses the impact of media awareness campaigns, the symptomatic population continues to grow and hence the infection will be maintained in the population.

In Figs. 13 and 14, the variation of virus density and symptomatic (hospitalized/isolated) infective population with time $t$ is shown for different values of $\theta$, the growth rate of viral density which is directly proportional to the asymptomatic infectives. It is seen from these figures that as the growth rate of viral density, which is proportional to the asymptomatic infectives, increases, the load of virus increases in the atmosphere (Fig. 13) and hence the symptomatic infectives population also increases (Fig. 14). Since the virus is directly emitted by the asymptomatic infective population and is deposited on surfaces/objects or remain airborne for some time, it increases the load of virus which ultimately results in increasing the symptomatic population. This suggests that if people are educated through media awareness campaigns to wear face cover/masks and avoid touching the infected surfaces/objects and maintain social distancing, the viral density in the atmosphere would decline. This decreased viral density will consequently make the symptomatic infectives population diminish. Thus, media awareness campaigns can be of vital importance to reduce the spreading of COVID-19 infection.

\section{Conclusion}

In this paper, a non-linear mathematical model is proposed and analyzed to study the effect of media awareness campaigns on the transmission dynamics of COVID-19 pandemic caused by the coronavirus SARS-CoV-2 in a population with variable size structure. It is assumed that the susceptibles become infected by direct contacts with asymptomatic infectives as well as by coming in contacts with virus deposited on surfaces and /or droplets which are airborne for some time due to coughing, sneezing, exhalation of symptomatic infectives, etc. In the proposed model, the total human population is divided into four sub-classes, namely susceptibles, asymptomatic infectives, aware 
susceptibles and symptomatic infectives (hospitalized/ isolated), incorporating classes representing cumulative density of virus and media alert. The model is analyzed using stability theory of differential equations and numerical simulation. Some inferences have been drawn regarding the spread of the disease by way of establishing local and global stability results. It is found that with increase in the dissemination of media awareness programs, the aware susceptible population increases and consequently the symptomatic infectives population decreases. Thus, if more and more susceptible persons become aware of disease escalation, the resulting behavioral change helps in reducing the spread of COVID-19 infection.

\section{Appendix 1}

Proof of Theorem 4.1. To prove this theorem, we have the following Jacobian matrix evaluated at $E_{0}$.

$$
\begin{aligned}
& J\left(E_{0}\right)= \\
& {\left[\begin{array}{cccccc}
-d & 0 & 0 & -\alpha_{1} & 0 & 0 \\
0 & {\left[\beta-\left(\alpha+d+\beta_{1}\right)\right]} & 0 & 0 & \lambda \frac{A}{d} & 0 \\
0 & 0 & -\left(\lambda_{11}+d\right) & 0 & 0 & 0 \\
0 & \beta_{1} & 0 & -\left(\alpha_{1}+d+\beta_{11}\right) & 0 & 0 \\
0 & \theta & 0 & 0 & -\theta_{0} & 0 \\
0 & \mu & 0 & 0 & 0 & -\sigma
\end{array}\right]}
\end{aligned}
$$

The characteristic equation is

$$
\begin{aligned}
g(x)= & \left(\lambda_{11}+d+x\right)\left(\alpha_{1}+d+\beta_{11}+x\right) \\
& (\sigma+x)\left(x^{3}+l_{1} x^{2}+l_{2} x+l_{3}\right)=0
\end{aligned}
$$

From Eq. (36) it is clear that the three eigen values are negative and remaining three can be checked by cubic equation, $\left(x^{3}+l_{1} x^{2}+l_{2} x+l_{3}\right)=0$, using Routh Hurwitz criteria

where, $l_{1}=d+\theta_{0}-\left[\beta-\left(\alpha+d+\beta_{1}\right)\right]$

$$
\begin{aligned}
& l_{2}=d \theta_{0}-\left[\beta-\left(\alpha+d+\beta_{1}\right)\right]\left(d+\theta_{0}\right) \\
& l_{3}=-\left[\beta-\left(\alpha+d+\beta_{1}\right)\right] d \theta_{0}-\lambda \frac{A}{d} \theta
\end{aligned}
$$

It can be easily seen that $l_{1}$ and $l_{2}$ are positive for $\beta<\left(\alpha+d+\beta_{1}\right)$, while $l_{3}$ is positive when $\beta+\lambda \frac{A \theta}{d \theta_{0}}<\left(\alpha+d+\beta_{1}\right)$. Therefore, only for a condition $\beta+\lambda \frac{A \theta}{d \theta_{0}}<\left(\alpha+d+\beta_{1}\right)$ the term $l_{1}, l_{2}$ and $l_{3}$ are positive and also satisfying $l_{1} l_{2}-l_{3}>0$. Which fulfills all the conditions of Routh Hurwitz criteria. Thus, the disease free equilibrium is locally asymptotically stable if $\beta+\lambda \frac{A \theta}{d \theta_{0}}<\left(\alpha+d+\beta_{1}\right)$ i.e $R_{0}<1$.

Proof of Theorem 4.2. Now to establish the local stability of the endemic equilibrium $E^{*}$, we linearize the system using small perturbations $n, y, x_{m}, y_{i}, v$ and $m$ as follows,

$N=N^{*}+n, Y=Y^{*}+y, X_{M}=X_{M}^{*}+x_{m}, Y_{i}=Y_{i}^{*}, V=V^{*}+$ $v, M=M^{*}+m$

Let us consider the following positive definite function,

$$
\begin{aligned}
U= & k_{1} \frac{1}{2} n^{2}+k_{2} \frac{1}{2} y^{2}+k_{3} \frac{1}{2} x_{m}^{2} \\
& +k_{4} \frac{1}{2} y_{i}^{2}+k_{5} \frac{1}{2} v^{2}+k_{6} \frac{1}{2} m^{2}
\end{aligned}
$$

where $k_{1}, k_{2}, k_{3}, k_{4}, k_{5}$ and $k_{6}$ are positive constants to be chosen appropriately.

On differentiating $U$ with respect to $t$

$$
\begin{aligned}
\frac{\mathrm{d} U}{\mathrm{~d} t}= & k_{1} n \frac{\mathrm{d} n}{\mathrm{~d} t}+k_{2} y \frac{\mathrm{d} y}{\mathrm{~d} t}+k_{3} x_{m} \frac{\mathrm{d} x_{m}}{\mathrm{~d} t} \\
& +k_{4} y_{i} \frac{\mathrm{d} y_{i}}{\mathrm{~d} t}+k_{5} v \frac{\mathrm{d} v}{\mathrm{~d} t}+k_{6} m \frac{\mathrm{d} m}{\mathrm{~d} t}
\end{aligned}
$$

Now using the linearized system of (7)-(12) and after some algebraic manipulations, we get

$$
\begin{aligned}
\frac{\mathrm{d} U}{\mathrm{~d} t}= & -k_{1} d n^{2}-k_{2} q y^{2}-k_{3}\left(a+\lambda_{11}+d\right) \\
& x_{m}^{2}-k_{4}\left(\alpha_{1}+d+\beta_{11}\right) y_{i}^{2}-k_{5} \theta_{0} v^{2}-k_{6} \sigma m^{2} \\
& +k_{2} p n y+k_{3} a n x_{m}-k_{1} \alpha_{1} n y_{i}-\left(k_{2} r+k_{3} a\right) y x_{m}+ \\
& \left(k_{4} \beta_{1}-k_{2} r\right) y y_{i} \\
& +\left(k_{5} \theta+k_{2} s\right) y v+k_{6} \mu y m-k_{3} a x_{m} y_{i}+k_{3} b x_{m} m
\end{aligned}
$$

Choosing, $k_{1}=1$, and after algebraic manipulation we o b t a i n $\quad k_{2}<\frac{4}{15} \frac{d q}{p^{2}}, \quad k_{3}<\min .\left(\phi_{1}, \phi_{2}, \phi_{3}, \phi_{4}\right)$, $\frac{9}{4} \frac{\alpha_{1}^{2}}{d\left(\alpha_{1}+d+\beta_{11}\right)}<k_{4}<\frac{4}{15} \frac{d q r}{p^{2} \beta_{1}}, k_{5}<\frac{4}{15} \frac{d q s}{p^{2} \theta}$ and $k_{6}<\frac{8}{75} \frac{d q^{2} \sigma}{p^{2} \mu^{2}}$.

Here $\phi_{1}=\frac{1}{3} \frac{d\left(a+\lambda_{11}+d\right)}{a^{2}}, \phi_{2}=\frac{4}{15} \frac{d q r}{p^{2} a}, \phi_{3}=\frac{4}{75} \frac{d q^{2} \sigma^{2}\left(a+\lambda_{11}+d\right)}{p^{2} \mu^{2} b^{2}}$, $\phi_{4}=\frac{1}{3} k_{4} \frac{\left(a+\lambda_{11}+d\right)\left(\alpha_{1}+d+\beta_{11}\right)}{a^{2}}$ we get $\frac{\mathrm{d} U}{\mathrm{~d} t}$ to be negative definite for the conditions given in the theorem.

\section{Appendix 2}

Proof of Theorem 4.3. To prove this theorem, we consider the following positive definite function,

$$
\begin{aligned}
W= & \frac{1}{2} p_{1}\left(N-N^{*}\right)^{2}+p_{2}\left(Y-Y^{*}-Y^{*} \ln \frac{Y}{Y^{*}}\right) \\
& +\frac{1}{2} p_{3}\left(X_{M}-X_{M}^{*}\right)^{2} \\
& +\frac{1}{2} p_{4}\left(Y_{i}-Y_{i}^{*}\right)^{2} \\
& +\frac{1}{2} p_{5}\left(V-V^{*}\right)^{2}+\frac{1}{2} p_{6}\left(M-M^{*}\right)^{2}
\end{aligned}
$$




$$
\begin{aligned}
\frac{\mathrm{d} W}{\mathrm{~d} t}= & p_{1}\left(N-N^{*}\right) \frac{\mathrm{d} N}{\mathrm{~d} t}+p_{2} \frac{\left(Y-Y^{*}\right)}{Y} \frac{\mathrm{d} Y}{\mathrm{~d} t} \\
& +p_{3}\left(X_{M}-X_{M}^{*}\right) \frac{\mathrm{d} X_{M}}{\mathrm{~d} t} \\
& +p_{4}\left(Y_{i}-Y_{i}^{*}\right) \frac{\mathrm{d} Y_{i}}{\mathrm{~d} t}+p_{5}\left(V-V^{*}\right) \\
& \frac{\mathrm{d} V}{\mathrm{~d} t}+p_{6}\left(M-M^{*}\right) \frac{\mathrm{d} M}{\mathrm{~d} t}
\end{aligned}
$$

where $p_{i}(i=1,2,3,4,5,6)$ are positive constants, to be chosen appropriately.

After some algebraic manipulations, $\frac{\mathrm{d} W}{\mathrm{~d} t}$ can be written as

$$
\begin{aligned}
\frac{\mathrm{d} W}{\mathrm{~d} t}= & -p_{1} d\left(N-N^{*}\right)^{2}-p_{2} \\
& {\left[\frac{r}{Y^{*}}+\lambda \frac{\left(N-Y-X_{M}-Y_{i}\right) V}{Y^{*} Y}\right]\left(Y-Y^{*}\right)^{2} } \\
& -p_{3}\left(a+\lambda_{11}+d\right)\left(X_{M}-X_{M}^{*}\right)^{2}-p_{4}\left(\alpha_{1}+d+\beta_{11}\right)\left(Y_{i}-Y_{i}^{*}\right)^{2} \\
& -p_{5} \theta_{0}(V-V *)^{2}-p_{6} \sigma\left(M-M^{*}\right)^{2}+p_{3} a\left(N-N^{*}\right)\left(X_{M}-X_{M}^{*}\right) \\
& +p_{2}\left[\frac{\beta\left(Y+X_{M}+Y_{i}\right)}{N N^{*}}+\lambda \frac{V^{*}}{Y^{*}}\right] \\
& \left(N-N^{*}\right)\left(Y-Y^{*}\right)-p_{1} \alpha_{1}\left(N-N^{*}\right)\left(Y_{i}-Y_{i}^{*}\right) \\
& -\left(p_{2} \frac{r}{Y^{*}}+p_{3} a\right)\left(Y-Y^{*}\right)\left(X_{M}-X_{M}^{*}\right) \\
& +\left(p_{4} \beta_{1}-p_{2} \frac{r}{Y^{*}}\right)\left(Y-Y^{*}\right)\left(Y_{i}-Y_{i}^{*}\right) \\
& +p_{6} \mu\left(Y-Y^{*}\right)\left(M-M^{*}\right)+p_{3} \frac{v\left(N-Y-X_{M}-Y_{i}\right)}{(1+\gamma M)\left(1+\gamma M^{*}\right)} \\
& \left(X_{M}-X_{M}^{*}\right)\left(M-M^{*}\right) \\
& +\left[p_{2} \frac{\lambda\left(N-Y-X_{M}-Y_{i}\right)}{Y^{*}}+p_{5} \theta\right]\left(Y-Y^{*}\right)\left(V-V^{*}\right) \\
& -p_{3} a\left(X_{M}-X_{M}^{*}\right)\left(Y_{i}-Y_{i}^{*}\right)
\end{aligned}
$$

The Eq. (42) can be rewritten as,

$$
\begin{aligned}
& \frac{\mathrm{d} W}{\mathrm{~d} t} \leq-p_{1} d\left(N-N^{*}\right)^{2}-p_{2} \\
& \frac{r}{Y^{*}}\left(Y-Y^{*}\right)^{2}-p_{3}\left(a+\lambda_{11}+d\right)\left(X_{M}-X_{M}^{*}\right)^{2} \\
& -p_{4}\left(\alpha_{1}+d+\beta_{11}\right)\left(Y_{i}-Y_{i}^{*}\right)^{2}-p_{5} \theta_{0}(V-V *)^{2}-p_{6} \sigma\left(M-M^{*}\right)^{2} \\
& +p_{2}\left[\frac{\beta}{N^{*}} \frac{\left(\alpha_{1}+d\right)}{d}+\lambda \frac{V^{*}}{Y^{*}}\right] \\
& \left(N-N^{*}\right)\left(Y-Y^{*}\right)+p_{3} a\left(N-N^{*}\right)\left(X_{M}-X_{M}^{*}\right) \\
& -p_{1} \alpha_{1}\left(N-N^{*}\right)\left(Y_{i}-Y_{i}^{*}\right)-\left(p_{2} \frac{r}{Y^{*}}+p_{3} a\right)\left(Y-Y^{*}\right)\left(X_{M}-X_{M}^{*}\right) \\
& +\left(p_{4} \beta_{1}-p_{2} \frac{r}{Y^{*}}\right)\left(Y-Y^{*}\right)\left(Y_{i}-Y_{i}^{*}\right)-p_{3} a\left(X_{M}-X_{M}^{*}\right)\left(Y_{i}-Y_{i}^{*}\right) \\
& +\left[p_{2} \frac{\lambda\left(\frac{A}{d}\right)}{Y^{*}}+p_{5} \theta\right]\left(Y-Y^{*}\right)\left(V-V^{*}\right)+p_{6} \mu\left(Y-Y^{*}\right)\left(M-M^{*}\right) \\
& +p_{3} \frac{v\left(\frac{A}{d}\right)}{\left(1+\gamma M^{*}\right)}\left(X_{M}-X_{M}^{*}\right)\left(M-M^{*}\right)
\end{aligned}
$$


Contreras S, Andres Villavicencio H, Medina-Ortiz D, Biron-Lattes JP, Olivera-Nappa A (2020) A multi-group SEIRA model for the spread of COVID-19 among heterogeneous populations. Chaos Solitons Fractals 136:109925

Cooper I, Mondal A, Antonopoulos CG (2020) A SIR model assumption for the spread of COVID-19 in different communities. Chaos Solitons Fractals 139:110057

Corbet S, Hou Y, Hu Y, Lucey B, Oxley L (2021) Aye Corona! The contagion effects of being named Corona during the COVID-19 pandemic. Finance Res Lett 38:101591

Cui J, Sun Y, Zhu H (2008a) The impact of media on the control of infectious disease. J Dyn Differ Equ 20(1):31-53

Cui J, Tao X, Zhu H (2008b) An SIS infection model incorporating media coverage. Rocky Mt J Math 38(5):1323-1334

Dubey B, Dubey V, Dubey US (2016) Role of media and treatment on an SIR model. Nonlinear Anal Model Control 21(2):185-200

Fredj HB, Cherif F (2020) Novel Corona virus disease infection in Tunisia: mathematical model and the impact of the quarantine strategy. Chaos Solitons Fractals 138:109969

Gao Y, Shi C, Chen Y, Shi P, Chen E (2020) A cluster of the Corona Virus Disease 2019 caused by incubation period transmission in Wuxi, China. J Infect 80(6):666-670

Hu Y, Sun J, Dai Z, Deng H, Xu Y (2020) Prevalence and severity of corona virus disease 2019 (COVID-19): a systematic review and meta-analysis. J Clin Virol 127:104371

Khan MA, Atangana A (2020) Modeling the dynamics of novel coronavirus (2019-nCov) with fractional derivative. Alex Eng J 59(4):2379-2389

Krishna VM, Prakash J (2020) Mathematical modelling on phase based transmissibility of Coronavirus. Infect Dis Model 5:375-385

Kumar A, Somani A (2020) Dealing with Corona virus anxiety and OCD. Asian J Psychiatry 51:102053

Liu Z, Magal P, Seydi O, Webb G (2020) A COVID-19 epidemic model with latency period. Infect Dis Model 5:323-337

Misra AK, Sharma A, Shukla JB (2011) Modeling and analysis of effects of awareness programs by media on the spread of infectious diseases. Math Comput Model 53:1221-1228

Misra AK, Sharma A, Shukla JB (2015) Stability analysis and optimal control of an epidemic model with awareness programs by media. Biosystems 138:53-62

Mitchel EP (2020) Corona Virus: global pandemic causing world-wide shutdown. J Natl Med Assoc 112(2):113-114

Naresh R, Tripathi A, Sharma D (2009) Modelling and analysis of the spread of AIDS epidemic with immigration of HIV infectives. Math Comput Model 49:880-892
Naresh R, Tripathi A, Sharma D (2011a) A nonlinear AIDS epidemic model with screening and time delay. Appl Math Comput 217:4416-4426

Naresh R, Tripathi A, Sharma D (2011b) A nonlinear HIV/AIDS model with contact tracing. Appl Math Comput 217:9575-9591

Pai C, Bhaskar A, Rawoot V (2020) Investigating the dynamics of COVID-19 pandemic in India under lockdown. Chaos Solitons Fractals 138:109988

Pandey SC, Pande V, Sati D, Upreti S, Samant M (2020) Vaccination strategies to combat novel corona virus SARS-CoV-2. Life Sci 2561:117956

Panovska-Griffiths J (2020) Can mathematical modelling solve the current COVID-19 crisis? BMC Public Health 20:551. https://doi. org/10.1186/s12889-020-08671-Z

Sarkar K, Khajanchi S, Nieto JJ (2020) Modeling and forecasting the COVID-19 pandemic in India. Chaos Solitons Fractals 139:110049

Shereen MA, Khan S, Kazmi A, Bashir N, Siddique R (2020) COVID19 infection: origin, transmission, and characteristics of human coronaviruses. J Adv Res 24:91-98

Tripathi A, Naresh R (2019) Modeling the role of media awareness programs on the spread of HIV/AIDS. World J Model Simul 15(1):12-24

Tripathi A, Naresh R, Sharma D (2007) Modelling the effect of screening of unaware infectives on the spread of HIV infection. Appl Math Comput 184:1053-1068

Wang D, Zhou M et al (2020) Epidemiological characteristics and transmission model of Corona Virus Disease 2019 in China. J Infect 80(5):e25-e27

Wiah EN, Danso-Addo E, Bentill DE (2020) Modelling the dynamics of COVID-19 disease with contact tracing and isolation in Ghana. Math Model Appl 5(3):146-155

(2021) www.covid19. who.int, Corona virus fact sheet by WHO

(2020) www.mygov.in/covid-19/, Corona virus statistics by India

Zeb A, Alzahrani E et al (2020) Mathematical model for Coronavirus Disease 2019 (COVID-19) containing isolation class. Biomed Res Int 3452402. https://doi.org/10.1155/2020/3452402

Zehra Z, Luthra M et al (2020) Corona virus versus existence of human on the earth: a computational and biophysical approach. Int J Biol Macromol 16115:271-281

Publisher's Note Springer Nature remains neutral with regard to jurisdictional claims in published maps and institutional affiliations. 